\title{
THE
}

\section{On the Geostrophic Adjustment of an Isolated Lens: Dependence on Burger Number and Initial Geometry}

\author{
Grant A. Stuart \\ Miles A. Sundermeyer \\ Dave Hebert \\ University of Rhode Island, davidhebert@uri.edu
}

Follow this and additional works at: https://digitalcommons.uri.edu/gsofacpubs

\section{Citation/Publisher Attribution}

Stuart, G. A., Sundermeyer, M. A., \& Hebert, D. (2011). On the Geostrophic Adjustment of an Isolated Lens: Dependence on Burger Number and Initial Geometry. Journal of Physical Oceanography, 41, 725-741. doi: 10.1175/2010JP04476.1

Available at: http://dx.doi.org/10.1175/2010JP04476.1

This Article is brought to you for free and open access by the Graduate School of Oceanography at DigitalCommons@URI. It has been accepted for inclusion in Graduate School of Oceanography Faculty Publications by an authorized administrator of DigitalCommons@URI. For more information, please contact digitalcommons-group@uri.edu. 


\title{
On the Geostrophic Adjustment of an Isolated Lens: Dependence on Burger Number and Initial Geometry*
}

\author{
Grant A. Stuart ${ }^{+}$And Miles A. Sundermeyer \\ University of Massachusetts Dartmouth, Dartmouth, Massachusetts \\ DAVE HEBERT ${ }^{\#}$ \\ Graduate School of Oceanography, University of Rhode Island, Narragansett, Rhode Island
}

(Manuscript received 1 April 2010, in final form 30 October 2010)

\begin{abstract}
Geostrophic adjustment of an isolated axisymmetric lens was examined to better understand the dependence of radial displacements and the adjusted velocity on the Burger number and the geometry of initial conditions. The behavior of the adjustment was examined using laboratory experiments and numerical simulations, which were in turn compared to published analytical solutions. Three defining length scales of the initial conditions were used to distinguish between various asymptotic behaviors for large and small Burger numbers: the Rossby radius of deformation, the horizontal length scale of the initial density defect, and the horizontal length scale of the initial pressure gradient. Numerical simulations for the fully nonlinear time-dependent adjustment agreed both qualitatively and quantitatively with analogous analytical solutions. For large Burger numbers, similar agreement was found in laboratory experiments. Results show that a broad range of final states can result from different initial geometries, depending on the values of the relevant length scales and the Burger number computed from initial conditions. For Burger numbers much larger or smaller than unity, differences between different initial geometries can readily exceed an order of magnitude for both displacement and velocity.
\end{abstract}

\section{Introduction}

\section{a. Motivation and background}

In classic geostrophic adjustment, an initially unbalanced state in the form of a front or lens is allowed to collapse under the combined influence of gravity and rotation. Through a combination of slumping and radiation of internal waves, a balanced or quasi-balanced flow is formed in which the pressure gradient is balanced by rotation and possibly nonlinear terms. In the textbook problem, the final state can be predicted from initial

\footnotetext{
* University of Massachusetts Dartmouth School for Marine Science and Technology Contribution Number 10-1102.

+ Current affiliation: Fugro Global Environmental \& Ocean Sciences, Houston, Texas.

\# Current affiliation: Fisheries and Oceans Canada, Bedford Institute of Oceanography, Dartmouth, Nova Scotia, Canada.
}

Corresponding author address: Miles A. Sundermeyer, School for Marine Science and Technology, University of Massachusetts Dartmouth, 706 S. Rodney French Blvd., New Bedford, MA 02744. E-mail: msundermeyer@umassd.edu parameters in terms of the Rossby radius of deformation, which is given by the distance a gravity wave will travel in one inertial period, and geostrophic velocity scaling, which represents a balance between the Coriolis and pressure gradient terms in the momentum equation.

When the scale of the adjusting density field is large compared to the deformation radius, the solution is generally considered to be well described in terms of such scaling. However, when the horizontal scale of the initial density anomaly is of the same order or less than the deformation radius, the adjustment is somewhat more complicated. In such cases, the adjustment process itself can generate order one changes in the horizontal and vertical scales of the initial pressure anomaly such that the parameters associated with the final state are significantly different than those associated with the initial state. The resultant flow may still be geostrophically balanced. However, the magnitude of the balanced flow will depend on a variety factors, including the scales and specific geometry of the initial density defect.

The general problem of geostrophic adjustment dates back to Rossby $(1937,1938)$ and has been well studied in the literature since then. Reviews of the classic adjustment 
problem, as well as many of its variants, may be found, for example, in Blumen (1972), Gill (1982), McWilliams (1985), and Flierl (1987). Subsequent studies have continued to relate the basic principles to a range of oceanographic phenomena, including the adjustment of density fronts and their associated meanders (e.g., Ou 1984; van Heijst 1985; Garvine 1987; Spall 1995; Blumen and Wu 1995), the ocean response to storms (e.g., Geisler 1970; Price 1981), the dynamics of dense water outflows (e.g., Price and O'Neil Baringer 1994; Cenedese et al. 2004), and ocean convection (e.g., Killworth 1979; Hermann and Owens 1993; Whitehead et al. 1996; Rubino et al. 2007), to name a few.

In the present study, we are interested in one subclass of the problem, the adjustment of an isolated lens in a continuously stratified fluid. This configuration can arise, for example, as the result of localized internal wave breaking in the ocean interior. Evidence of patchy mixing in the ocean has been reported by numerous investigators (e.g., Grant et al. 1968; Woods and Wiley 1972; Gregg 1980; Gregg et al. 1986; Alford and Pinkel 2000; Oakey and Greenan 2004; Sundermeyer et al. 2005). Such localized mixing can lead to gravitational adjustment of the mixed regions, which, under the influence of rotation, can in turn lead to small-scale geostrophically balanced flows (e.g., Garrett and Munk 1972; McWilliams 1985). These small-scale balanced motions have been referred to in the literature as the vortical mode, semipermanent fine structure, pancake eddies, or blini (e.g., Kunze 2001; Polzin et al. 2003). In practice, they have aspect ratios of order $f / N$, similar to the internal wave field, with scales ranging from hundreds of meters to many kilometers horizontally and on the order of meters to tens of meters vertically. As described by Sundermeyer et al. (2005) and Sundermeyer and Lelong (2005), the motions from many of these adjustments can also contribute significantly to submesoscale lateral dispersion in the ocean interior. It is the latter that motivates our particular interest in the displacements generated by such eddies.

In addition to localized internal wave breaking, numerous other processes have been hypothesized to contribute to vortical mode energy in the ocean. These include the downscale transfer of variance associated with the potential enstrophy cascade of geostrophic turbulence (e.g., Charney 1971), detrainment and subduction of surface mixed layer water (e.g., Stommel 1979; Marshall et al. 1993; Spall 1995), and intensified mixing near topography (e.g., D'Asaro 1988; Kunze and Sanford 1993). In the present context, however, we restrict our attention to the geometry most appropriate to the adjustment of mixed patches generated by diapycnal mixing events. For this generation mechanism, the so-called s vortex described by Morel and McWilliams (1997) is particularly appropriate. Such a vortex is formed when a localized region of reduced stratification is allowed to adjust under the influence of rotation to form a core anticyclone (associated with vortex compression at the center of the mixed region), sandwiched above and below by two weaker cyclones (associated with vortex stretching above and below the main mixed region).

Other vortex geometries (e.g., without the accompanying cyclones above and below, of varying steepness, and/or isolated versus nonisolated) have also been used to model oceanic eddies in a variety of contexts and scales, including Mediterranean eddies (e.g., Armi and Zenk 1984; Hebert et al. 1990), Gulf Stream warm-core rings (e.g., Saunders 1971; Robinson et al. 1988; Olson 1991), submesoscale coherent vortices found both in the open ocean and in coastal waters (e.g., McWilliams 1985; D'Asaro 1988; Dewar and Killworth 1990), and the order $100 \mathrm{~m}$ to a few kilometer submesoscale eddies envisioned here.

Analytical solutions under varying conditions have also been discussed in the literature (e.g., Csanady 1979; Flierl 1979; Ou 1986; McWilliams 1988, hereafter M88; Killworth 1992; Boss and Thompson 1995; Spall 1995; Kuo and Polvani 1997; Ungarish and Huppert 1998; Reznik et al. 2001; Dotsenko and Rubino 2006), and a number of these are considered in more detail below. For the purposes of the present study, we will focus in particular on the semianalytical solutions of M88 for the adjustment of an axisymmetric lens in a continuously stratified rotating fluid, which is also discussed below. Time-dependent numerical solutions of this same configuration were discussed by Lelong and Sundermeyer (2005) and will also be revisited here.

The problem of geostrophic adjustment has also been studied in a variety of forms in the laboratory. Relevant to the present study, Saunders (1973) as well as Rubino and Brandt (2003) conducted experiments to test the simplest form of geostrophic adjustment in which they removed a thin-walled cylinder that separated higher and lower density fluids in a rotating cylindrical tank. Stegner et al. (2004) conducted similar two-layer adjustment experiments, but with a separate shallow layer of less dense water and without extending the cylinder walls to the tank bottom. Hedstrom and Armi (1988) studied the geostrophic adjustment of homogeneous density patches within a linearly stratified, rotating background. In their study, the density patches were formed by injecting fluid volumes at middepth. Numerous laboratory studies have also examined the behavior of isolated vortices in various fluid environments, ignoring the dynamics of eddy formation. An extensive review of the behavior of vortices in rotating fluids was provided by Hopfinger and van Heijst (1993). Finally, although not 
in a rotating environment, experiments examining collapsing turbulent regions were studied by De Silva and Fernando (1998). Although they did not specifically study the problem of geostrophic adjustment, we note their work here because the experimental technique used in their study was similar to the present study.

\section{b. Scope and outline}

Among the many theoretical, numerical, and laboratory studies of geostrophic adjustment, a consistent finding is that both the velocity and length scales of the adjusted state depend on the value of a key nondimensional parameter, the Burger number $(\mathrm{Bu})$. However, the details of this dependence vary from one study to another, depending on the exact geometry of the problem examined. In the present study we revisit the problem of the geostrophic adjustment of an isolated lens in a rotating stratified fluid with an eye toward understanding this dependence and why it arises. As discussed above, the motivation for our interest in this particular problem is the effect of small-scale geostrophic motions on lateral dispersion. However, we believe the problem is also of more general interest. In section 2, we review selected analytical solutions by previous authors, with emphasis on the adjustment length and velocity scales and the behavior of various analytical solutions in the limits of small and large Burger numbers. In sections 3 and 4 , numerical and laboratory results, respectively, for the adjustment of an isolated lens in a continuously stratified fluid are compared with theoretical solutions. Implications for submesoscale coherent vortices in terms of generation scales and their final balanced states are discussed in section 5. Section 6 summarizes and concludes.

\section{Theoretical background}

\section{a. A progression of adjustment solutions}

Consider the problem of a density/pressure anomaly, initially at rest, which is allowed to relax under the influence of rotation, ultimately to a geostrophically balanced state. To better understand how the geometry of the initial condition affects the final state, we begin by examining a progression of analytical adjustment solutions found in the literature, with initial conditions ranging from a two-layer two-dimensional (2D) step function, to an axisymmetric lens in a three-dimensional continuously stratified fluid. Specifically, we revisit the basic two-dimensional dam break problem described in many oceanography textbooks (e.g., Gill 1982; Cushman-Roisin 1994), the axisymmetric two-layer "top hat" solutions of Csanady (1979, linear case) and Flierl (1979, nonlinear case) and the two- and three-dimensional five-layer and continuously stratified nonlinear adjustment solutions of
M88. Recognizing that analytical solutions for numerous other configurations exist in the literature (e.g., see references cited above), we choose these solutions because their particular geometries highlight key aspects of the problem. Of particular interest here is the behavior of each of these solutions in terms of the radial displacements generated during the adjustment and the maximum velocity of the adjusted state. For all solutions considered here, the initial condition is a state of rest. Key features of the different geometries are depicted schematically in Fig. 1.

Before we begin, it is useful to distinguish a number of length scales relative to the geostrophic adjustment problem. First, regarding the initial state of the density anomaly, we define the initial horizontal length scale of the density defect $L_{d}$ (e.g., the radius of the initial density anomaly). Second, we define the horizontal length scale of the pressure gradient $L_{p}$, which may or may not equal $L_{d}$. For example, for the top-hat initial conditions of Csanady (1979) and Flierl (1979), although the length scale of the density defect $L_{d}$ is nonzero, the length scale of the initial pressure gradient $L_{p}$ is zero, because in this case the density is a step function. By contrast, for the parabolic lens initial condition of M88, the two length scales are comparable in size, because the pressure varies gradually from the center to the edge of the anomaly. Third, we define the traditional Rossby radius of deformation, either as $R=\sqrt{g^{\prime} h} / f$ for layered configurations or analogously as $R=N h / f$ for continuously stratified cases, where $g^{\prime}$ is reduced gravity based on the density difference between the two layers; $f$ is the Coriolis parameter; and $h$ and $N$ are the initial height and stratification, respectively. Note that, for each of the three length scales defined in the initial condition, there are also corresponding final length scales associated with the adjusted state. In general, these final length scales may be different from the initial values.

With the above length scales in mind, we pose the following question: given an initial density/pressure anomaly, under what conditions is there a simple relationship between the final state of the adjustment and initial parameters of the problem? The answer depends on the value of the Burger number, $\mathrm{Bu}=R^{2} / L^{2}$ : that is, the ratio of the deformation radius to the inherent length scale of the problem. Classically, for small $\mathrm{Bu}$, the adjustment reverts more or less to geostrophic scaling so that the final state is well predicted by the initial parameters of the problem. However, for $\mathrm{Bu}$ of order 1 or greater, significant deformations of the initial density/pressure field can occur, and the problem becomes more complex. To better understand both the limits of large and small $\mathrm{Bu}$, as well as the behavior at intermediate $\mathrm{Bu}$, we now revisit the above referenced analytical solutions with 
(a)

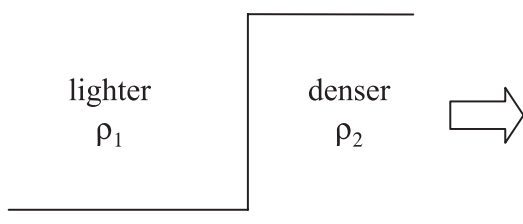

(b)
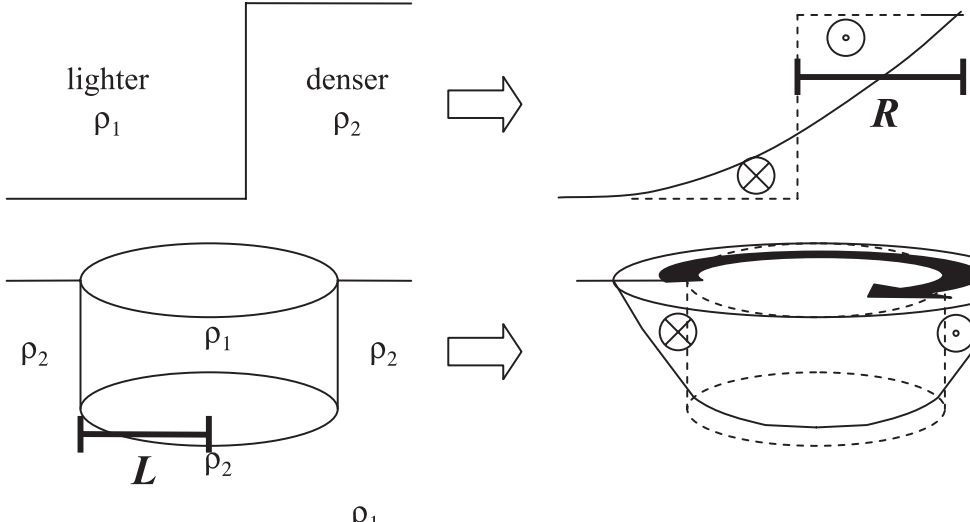

(c)
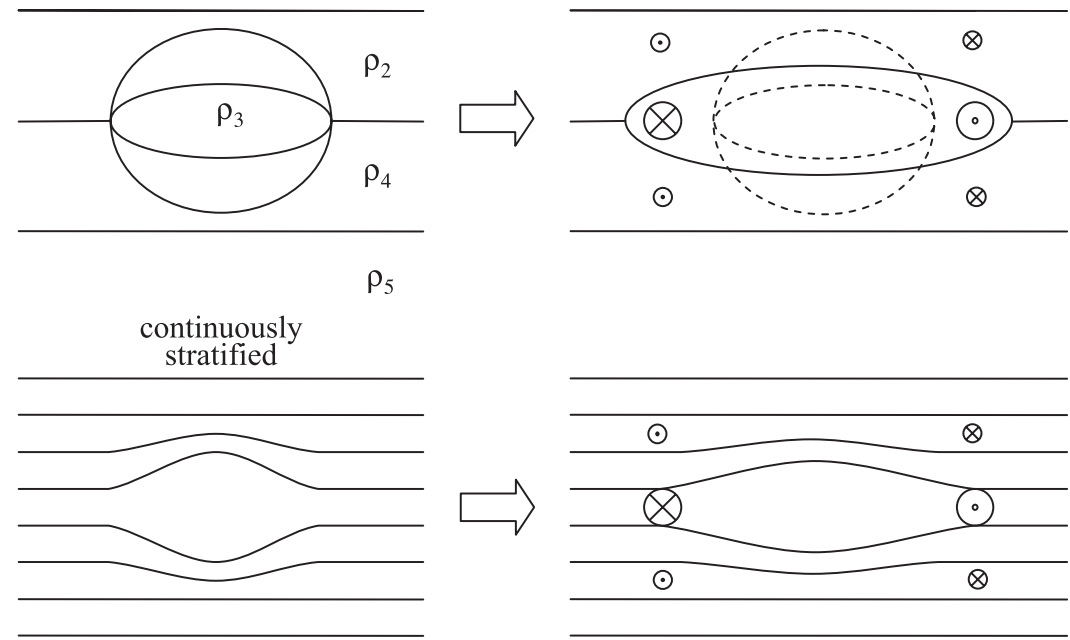

FIG. 1. Progression of geostrophic adjustment configurations considered in the present study, after (a) Gill (1982), (b) Csanady (1979) and Flierl (1979), and (c),(d) M88.

an eye toward the understanding their Burger number dependence. These solutions are then used to interpret the behavior of numerical simulations and laboratory experiments of the adjustment of an isolated lens.

\section{b. Burger number dependence}

For the geostrophic adjustment problem envisioned here, the Burger number can be thought of as an indicator of whether, based on its initial length scale, $L$, a horizontal density defect is likely to feel the effects of rotation during gravitational collapse. For $\mathrm{Bu} \ll 1, L$ is large compared to $R$ and the adjustment is strongly constrained by rotation. For $\mathrm{Bu} \gg 1$, the opposite is true: rotation is negligible and the motion is dominantly down pressure gradient, presumably either as an accelerating flow or balanced ultimately by friction. For $\mathrm{Bu} \sim O(1)$, the problem is more complex: initially, the density defect may be of small enough horizontal scale to not be significantly influenced by rotation, but as it slumps under gravity its horizontal scale increases. Even if $\mathrm{Bu}>1$ initially, if frictional effects are small, Bu may approach unity during the adjustment such that ultimately the adjustment feels the effect of rotation.

In addition to the Burger number dependence, the precise behavior of adjustment, both in terms of the degree of slumping that occurs before the pressure gradient is balanced by rotation and in terms of the ensuing geostrophically balanced flow, will also depend on the geometry of the initial condition. This can be illustrated by considering the above adjustment solutions in terms of two metrics: the difference between the initial and final horizontal length scales of the density defect $\Delta L=$ $L_{d_{f}}-L_{d_{i}}$ and the maximum geostrophically balanced velocity $U_{\max }$ generated by the adjustment. Noteworthy here is that, thus far, we have not defined which of the two horizontal length scales, $L_{d}$ or $L_{p}$, is the correct one to use in the Burger number. Because the pressure gradient force is what ultimately balances the Coriolis force, formally $L$ should be given by $L_{p}$. However, for layer solutions involving step functions in the initial density 


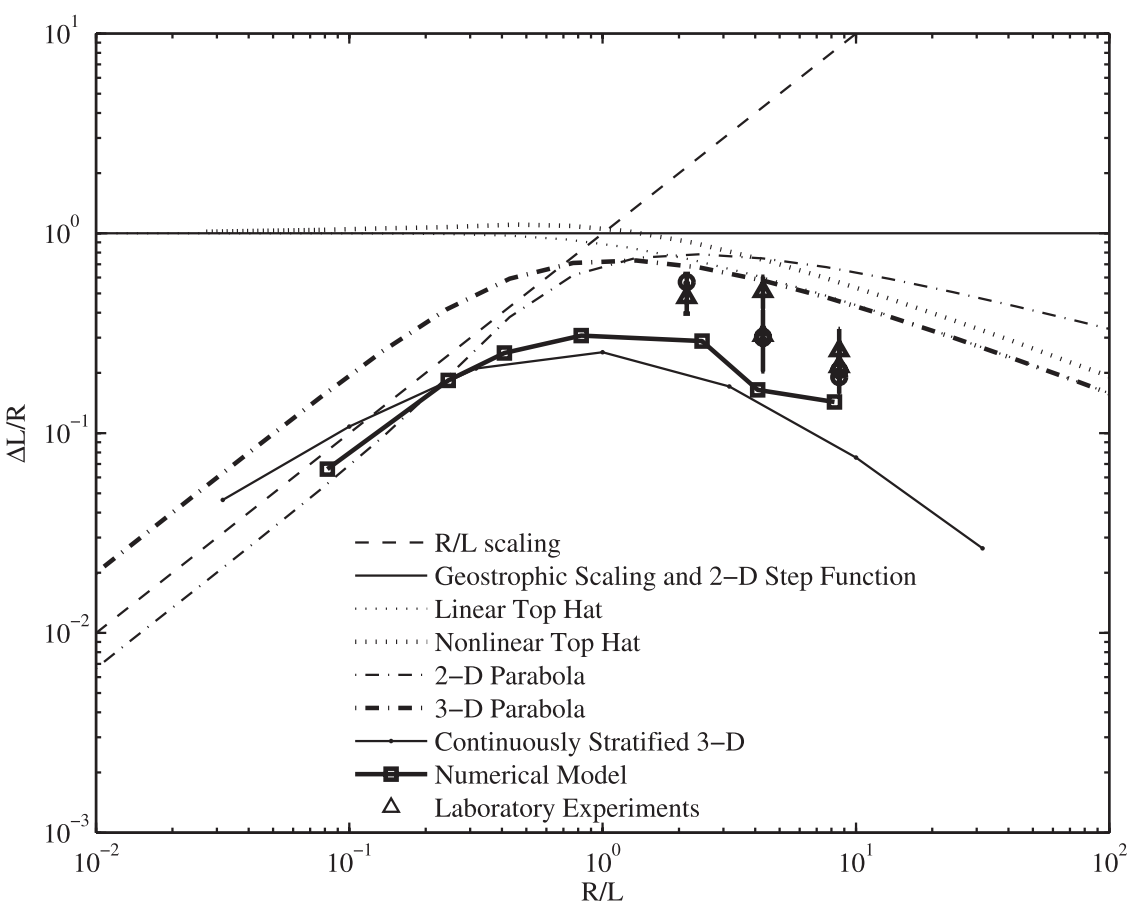

FIG. 2. Normalized adjustment distance plotted $v \mathrm{Bu}^{1 / 2}=R / L$ for various analytical, numerical, and laboratory results discussed in the text. Data from laboratory experiments based on both dye (triangles) and PIV (circles) are also shown.

distribution, $L_{d}$ is frequently used, because in those cases $L_{p}=0$. Because the initial conditions discussed here have $L_{d}=L_{p}$ whenever $L_{p}$ is nonzero, henceforth we shall assume that the $L$ (without subscript) is given by $L_{d}$. Also, in the discussion that follows, we shall nondimensionalize $\Delta L$ by $R$ and $U_{\max }$ by $f L$.

The Burger number dependence of the adjustment distance and maximum geostrophic velocity for each of the above analytical solutions is shown in Figs. 2 and 3, respectively. Considering the simplest initial condition first, the dam break problem described by Gill (1982), the initial density is a step function such that the initial pressure gradient is a delta function. The horizontal scale of the pressure gradient $L_{p}$ is thus zero, whereas the horizontal scale of the density defect $L_{d}$ is infinite. The Burger number, defined in terms of the scale of the density defect is therefore zero. In this limiting case, the scale over which the adjustment occurs is given exactly by the deformation radius $R=\sqrt{g^{\prime} h} / f$. The value of $\Delta L$ is thus exactly equal to $R$. Meanwhile, the maximum geostrophically balanced velocity is $U_{\max }=\sqrt{g^{\prime} h}$. Although $R / L$ is formally infinite for this solution, $\Delta L / R$ is well defined and equal to 1 for all $R$. We can thus represent this solution as unity on a $\Delta L / R$ versus $R / L$ plot (Fig. 2). Similarly, $U_{\max }$ normalized by $f L$ yields $R / L$, giving a line of slope 1 on a $U_{\max } / f L$ versus $R / L$ plot (Fig. 3).
Considering next the top-hat solutions of Csanady (1979) and Flierl (1979), again the initial density is a step function. However, this time the horizontal scale of the density defect $L_{d}$ is finite. In this case, $\mathrm{Bu}$ and hence the final solution for $\Delta L$ depend on the initial scale of the density defect $L_{d}$. For $\mathrm{Bu} \ll 1$ (i.e., $L \gg R$ ), the geometry approaches that of Gill (1982) and the adjustment distance $\Delta L$ approaches $R$ (Fig. 2). For $\mathrm{Bu} \gg 1$, however, the adjustment distance $\Delta L$ becomes much less than $R$ estimated from the initial condition. This is because the finite volume of the density defect leads to a decrease in $h$ as the density slumps during adjustment, which in turn reduces the effective $R$ compared to its initial value. For $\mathrm{Bu}>1$, the result is a tailing off of $\Delta L / R$ compared to the small Bu limit of unity. Similarly for $U_{\max }$, the solution of Csanady (1979) and Flierl (1979) both approach Gill's (1982) solution in the limit of $\mathrm{Bu} \ll 1$, whereas for $\mathrm{Bu}>1$ their solutions are again less than Gill's $U_{\max }=\sqrt{g^{\prime} h}$ (Fig. 3). The reason for this can again be understood in terms of the decrease in $h$ and increase in $L$ during the adjustment, both of which contribute to a smaller pressure gradient and hence smaller velocity associated with the balanced state.

Turning to M88's solutions, his five-layer solutions behave somewhat analogous to those of Csanady (1979) and Flierl (1979), except that this time the initial condition 


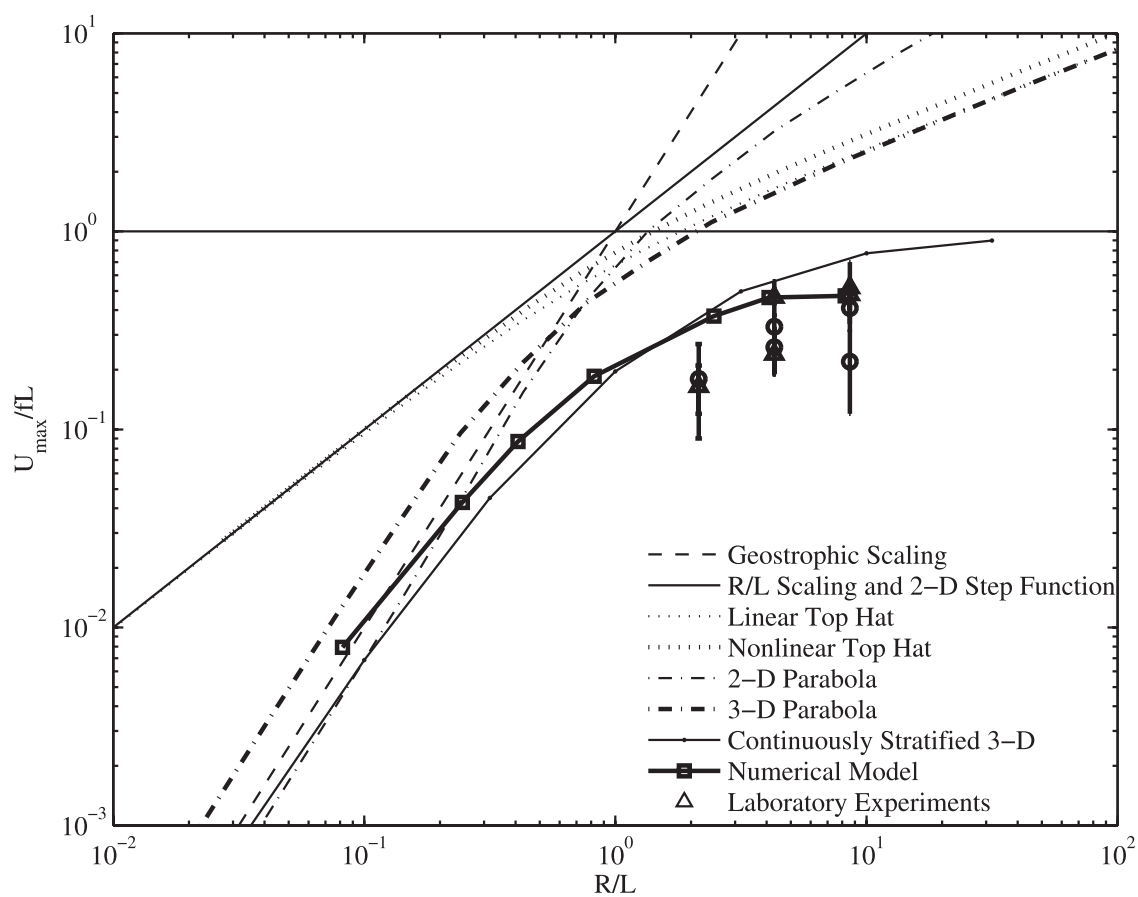

FIG. 3. Normalized maximum adjusted velocity $U_{\max }$ plotted vs $\mathrm{Bu}^{1 / 2}=R / L$ for various analytical, numerical, and laboratory results discussed in the text. Data from laboratory experiments based on both dye (triangles) and PIV (circles) are also shown. A line for normalized $U_{\max }=f L$ (i.e., Rossby number $=1$ ) is also shown for reference.

is parabolic rather than a step function, which results in a nonzero pressure gradient length scale $L_{p}$. For $\mathrm{Bu} \gg 1$, both the two- and three-dimensional solutions of M88 have an adjustment distance $\Delta L$ that behaves similarly to the Csanady (1979) and Flierl (1979) solutions, although they differ slightly in magnitude (Fig. 2). This again highlights the fact that, for large $\mathrm{Bu}$, the decrease in $h$ caused by slumping results in an adjustment that never reaches the deformation scale $R$, as determined from the initial conditions. For $\mathrm{Bu} \sim O(1)$, the solutions for $\Delta L$ and $U_{\max }$ both approach the result of Gill (1982) (see also Fig. 3). However, for $\mathrm{Bu} \ll 1$, the M88 solutions for $\Delta L$ and $U_{\max }$ are smaller than the previous solutions. This is because, in M88's parabolic initial condition, when $L_{d}$ is large, $L_{p}$ is also large. As such, for the same density difference $\Delta \rho$ between the respective layers, the pressure gradient and hence the resulting displacement $\Delta L$ and adjusted velocity $U_{\max }$ are both smaller compared to the analogous step function initial condition. Noteworthy here is that, for $\mathrm{Bu} \ll 1$, the adjusted velocity scales as $U_{\max } / f L \sim R^{2} / L^{2}$ (i.e., a slope of 2 on the normalized $U_{\max }$ versus $R / L$ plot of Fig. 3 ), consistent with pressure gradient scaling. Furthermore, the adjustment distance approaches $\Delta L / R \sim R / L$. The latter can be interpreted as the advective length scale associated with this $U_{\max }$ acting over a time scale $1 / f$ : that is, the canonical "distance a gravity wave will travel over one inertial period."

Finally, considering M88's continuously stratified solution, we find similar dependence on $\mathrm{Bu}$ for both $\Delta L$ and $U_{\max }$ compared to his parabolic layer solutions, although again they differ slightly in magnitude from the previous results. Also, for this case, as noted above, the deformation radius and the normalization for $U_{\max }$ are given in terms of their continuously stratified analogs.

\section{Results from numerical simulations}

\section{a. Numerical model setup}

As confirmation of the analytical solutions above and to complement the laboratory experiments to follow, we next examine results from numerical simulations of the adjustment of a continuously stratified lens based on a three-dimensional numerical model. The geometry of the initial stratification anomaly is the same as in the continuously stratified analytical solution of M88. However, here the numerical solutions are for the fully nonlinear time-dependent geostrophic adjustment problem, after Lelong and Sundermeyer (2005) and Sundermeyer and Lelong (2005). The simulations use the numerical model of Winters et al. (2004) to solve the threedimensional $f$-plane Boussinesq equations. The model 
equations were solved spectrally on a triply periodic domain via

$$
\begin{aligned}
\frac{D \mathbf{u}}{D t}+f \mathbf{i}_{3} \times \mathbf{u} & =-\frac{1}{\rho_{o}} \nabla P-\mathbf{i}_{3} \frac{g}{\rho_{o}} \rho+\nu_{6} \nabla^{6} \mathbf{u}, \\
\frac{D \rho}{D t} & =\kappa_{6} \nabla^{6} \rho, \text { and } \\
\nabla \cdot \mathbf{u} & =0,
\end{aligned}
$$

where all variables have their traditional meanings. Additional details regarding the model can be found in the Lelong and Sundermeyer (2005) and Sundermeyer and Lelong (2005) and will not be reviewed here.

Simulations were run using 64 grid points in the vertical by $128 \times 128$ grid points in the horizontal. Following Lelong and Sundermeyer (2005), in all runs, the Coriolis frequency was increased by a factor of 10 relative to realistic values. This reduced the ratio of the buoyancy frequency to the Coriolis frequency $N / f$ from a realistic value of approximately 200 to a more tractable value of approximately 20 . This allowed us to capture the dynamics associated with both of these time scales (i.e., internal waves and geostrophic adjustment) without having to perform prohibitively long numerical integrations or use prohibitively small time steps. Horizontal and vertical domain sizes for the base case were $L_{x}, L_{y}=$ $500 \mathrm{~m}$ (equivalently $L_{x}, L_{y}=5000 \mathrm{~m}$ after $N / f$ scaling) and $L_{z}=12.5 \mathrm{~m}$, respectively. Finally, our runs were effectively inviscid, with the exception of a hyperviscosity that removed energy at the smallest scales.

In all cases, the initial condition in the model was a state of rest and uniform stratification, superimposed by a Gaussian-shaped stratification anomaly similar to the three-dimensional continuously stratified analytical form used by M88. From this initial condition, the stratification anomaly was allowed to freely adjust to form a geostrophically balanced eddy plus internal waves. Noteworthy here is that, in general, the partition of energy between the balanced vortex and radiated waves will depend not only on the Burger number but also on the initialization procedure used to create the vortex, as discussed, for example, by Dritschel and Viúdez (2007). Relating the present initialization to theirs, we effectively generate our anomalies instantaneously. The kinetic and potential energy partition between the wave and vortex for this configuration is described in detail by Lelong and Sundermeyer (2005).

Analysis of the numerical solutions in terms of the initial and final length scales was done by fitting a Gaussian to the stratification anomaly at the vertical center of the mixed patch and taking the horizontal $e$-folding scale both from the prescribed initial condition $\left(L_{d_{i}}\right)$ and averaged between 2 and 3 inertial periods following release of the mixed patch $\left(L_{d_{f}}\right)$. Similarly, the adjusted velocity scale was taken as the maximum azimuthal velocity averaged over the same time period. The delay of 2 inertial periods rather than using the first or second inertial period avoided the largest transients in the velocity. Averaging over 1 complete inertial period limited contamination by any transients that remained. Note that, because our model is triply periodic, waves that radiate away during the initial adjustment re-enter the domain after as little as a few inertial periods. We have performed simulations with larger domain sizes and found that the dynamics of the adjustment and the above metrics for the eventual balanced vortex are not particularly sensitive to domain size and hence the re-entry of waves (see also Lelong and Sundermeyer 2005). This finding is supported by previous studies suggesting that interaction between waves (particularly higher-frequency waves) and the geostrophic component are likely weak (e.g., Bartello 1995; Dritschel and Viúdez 2007).

\section{b. Burger number dependence}

The difference between the initial and final length scales for the density defect $\Delta L$ and the maximum azimuthal velocity $U_{\max }$ of the numerical solutions for a range of Burger numbers are overplotted in Figs. 2 and 3, respectively. The results have been normalized in the same manner as the analytical solutions. Considering both $\Delta L$ and $U_{\max }$ and allowing for a constant scale factor, the numerical solutions show good agreement with analogous analytical solutions by M88 for a continuously stratified axisymmetric lens for the range of Bu examined. As with the continuously stratified analytical solutions, $\Delta L$ and $U_{\max }$ for the numerical results are consistently lower than the other analytical cases for $\mathrm{Bu} \geq 1$. However, $U_{\max }$ approaches geostrophic scaling and $\Delta L / R$ approaches $R / L$ for small $\mathrm{Bu}$. The numerical results are thus consistent with the idea that the behavior for small $\mathrm{Bu}$ is the result of the nonzero length scale associated with the pressure gradient, whereas the rolloff at large $\mathrm{Bu}$ is due to volumetric effects associated with the initial slumping of the density defect.

\section{Results from laboratory experiments}

Using the above analytical and numerical simulations as a baseline for understanding the Burger number dependence of the adjustment problem, we next examine laboratory experiments of the adjustment of an isolated lens in a continuously stratified rotating fluid. Again, our experiments are performed having in mind spatial scales associated with the problem of mixed patches created by internal wave breaking in the ocean. We reiterate, 


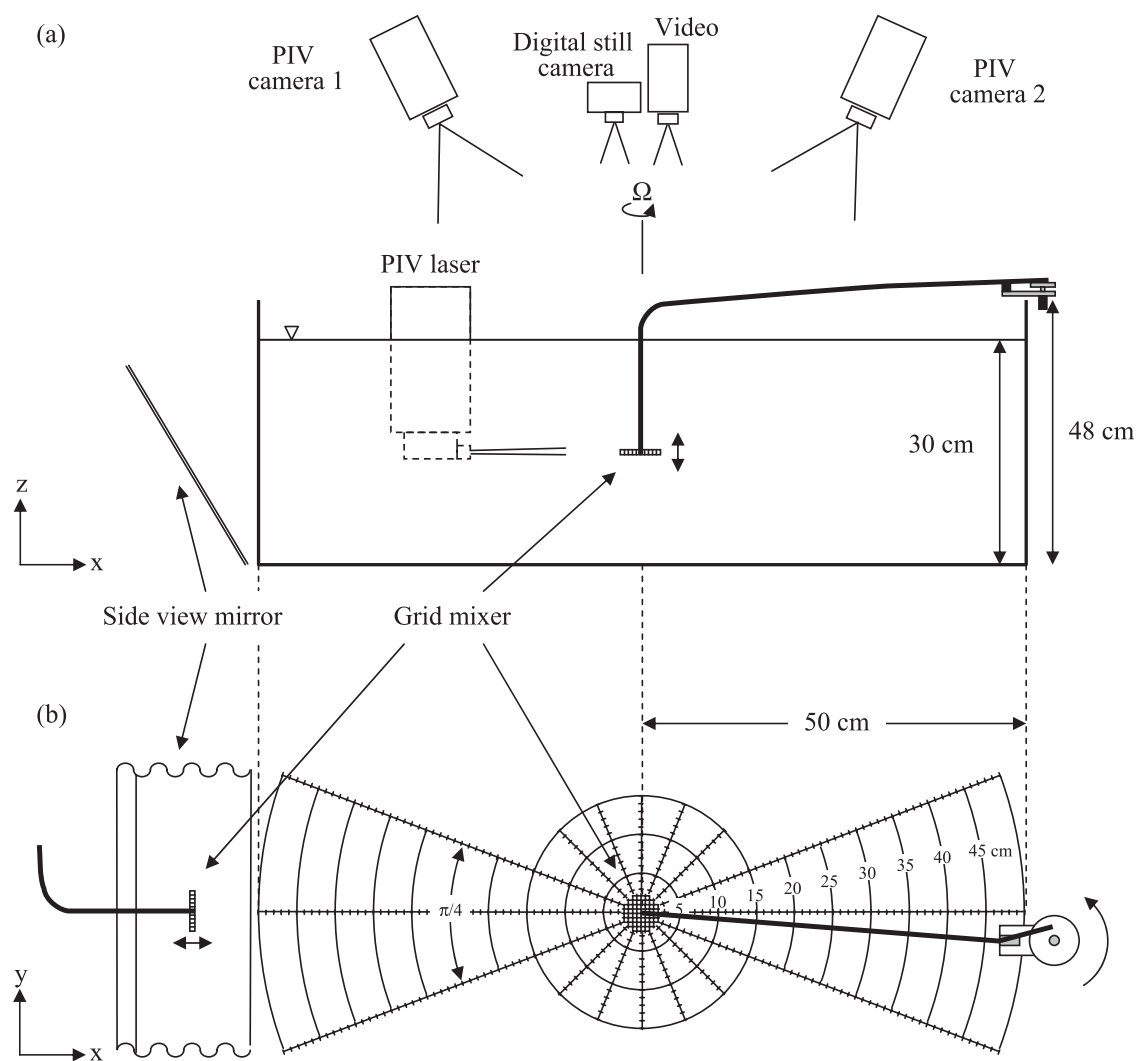

FIG. 4. Tank schematic for laboratory experiments showing (a) vertical cross-sectional and (b) plan views.

however, that, because the problem is readily nondimensionalized, the results can be easily generalized to larger-scale contexts. In what follows, we also note that because of limitations related to the size of the experimental setup, the laboratory results presented are limited to Burger numbers greater than one.

\section{a. Experimental setup}

Laboratory experiments were conducted on a highprecision rotating table, manufactured by Australian Scientific Instruments (Australian Scientific Instruments 2010) and housed at the Geophysical Fluid Dynamics laboratory of the University of Rhode Island's Graduate School of Oceanography. The experimental tank used in all experiments was a cylindrical acrylic tank with a diameter of $1.0 \mathrm{~m}$ (Fig. 4). To correct for optical distortions when viewing from the side, the cylindrical tank was enclosed by a larger square acrylic tank. The volume between the two tanks was filled with fluid of the same density and stratification. A single, angled flat mirror at the perimeter of the tank allowed overhead cameras to photograph both plan and side views simultaneously. An acrylic lid was used to eliminate surface stress effects on the fluid.
All experiments were conducted with a linearly stratified salt solution using the two-tank method (Fortuin 1960). The buoyancy frequency $N$ of the linearly saltstratified tank was predetermined based on the initial densities of the two tanks. This was also verified by measuring the density of fluid samples drawn from the bottom and top of the experiment tank using a refractometer. To ensure there were no vertical temperature gradients, the salt fluid was equilibrated overnight to ambient room temperature. This also allowed it to de-gas prior to each experiment. The table rotated counterclockwise as viewed from above, with various rotation rates set for different experiments. The depth of the fluid for all experiments was $30 \mathrm{~cm}$.

Localized mixed regions of fluid were formed by vertically oscillating a horizontal grid positioned at middepth $(z=15 \mathrm{~cm}$, Fig. 4$)$. The stainless steel mesh grid was a $5.1-\mathrm{cm}$-diameter circular disk painted with a mixture of black and rhodamine WT fluorescent dye to minimize specular reflection by the laser during particle image velocimetry (PIV) visualization. A slider-crank mechanism was used to oscillate the mixing grids through a vertical stroke distance of $3.0 \mathrm{~cm}$ at a frequency of approximately $1.0 \mathrm{~Hz}$. The mixer was activated for $25 \mathrm{~s}$ 


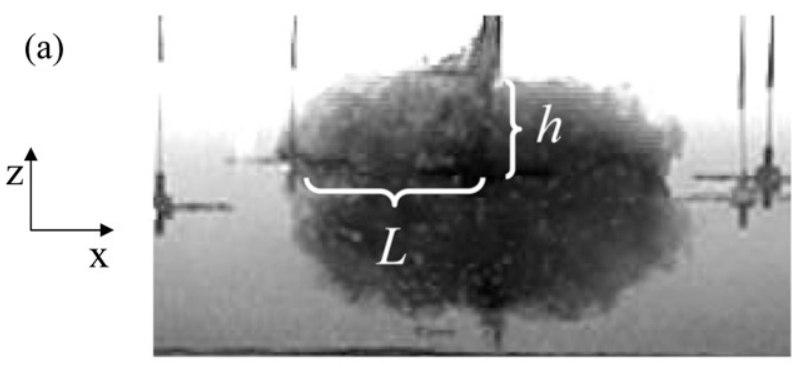

(b)

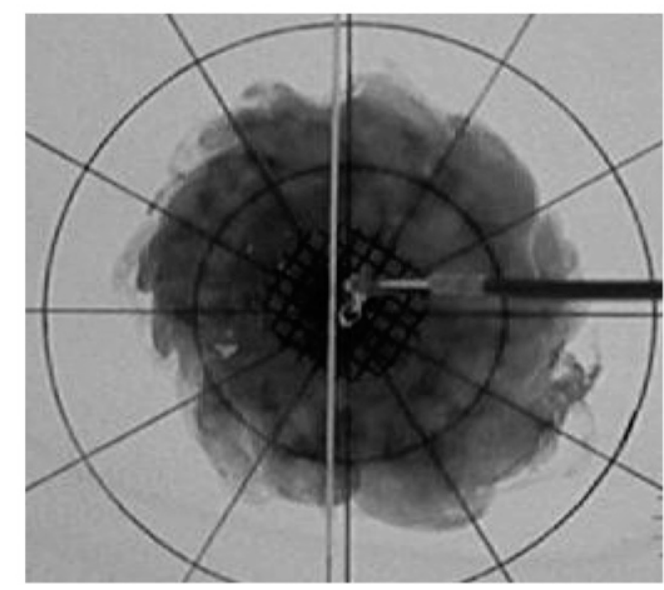

FIG. 5. Example of geostrophically adjusted eddy for base case laboratory experiment. (a) Side view of density anomaly stained by dye. The parameters $h$ and $L$ are indicated. (b) Plan view of same eddy. Note for scale that the diameter of the mixer is $5.08 \mathrm{~cm}$ and the concentric circles on the bottom of the tank are at $5-\mathrm{cm}$ in tervals.

to ensure that the mixed patch was thoroughly mixed: that is, $N_{\text {patch }}=0$. This allowed the difference in stratification $\Delta N$ between the mixed patch and the stratified background (i.e., $\Delta N=N-N_{\text {patch }}$ ) to equal the value of the buoyancy frequency $N$. A wireless, remote PC control using LabView software and a National Instruments Fieldpoint relay module enabled automated start/stop function for consistency between experiments. An example of both plan and side views of a mixed patch, visualized using dye, is shown in Fig. 5.

\section{b. $\Delta L$ and $U_{\text {max }}$ from dye measurements}

A passive tracer was used to visualize the adjustment and to estimate the radial displacement and eddy velocity for 5 of a total of 10 adjustment experiments examined. For each of these experiments, dye was mixed with an appropriate ratio of ethyl alcohol (e.g., 20:1 for $N=0.86 \mathrm{rad} \mathrm{s}^{-1}$ using $91 \%$ ethanol solution) to make the dye neutrally buoyant at the nominal middepth of the mixed patch. A syringe pump injected the dye at $1 \mathrm{~mL} \min ^{-1}$ for 3 table rotations at the equilibrium depth at the center of the mixer. A digital camera was used to take high-resolution images of the dye during the experiments. The evolution of the dye patch was also recorded continuously using a digital video camcorder.

A series of images taken within the first few inertial periods of the adjustment for the base case dye run is shown in Fig. 6. These reveal the horizontal adjustment and spinup of an anticyclonic vortex. The growing dye patch, as well as dye wisps visible around its perimeter, shows the slumping and spinup of the adjusted eddy. From these dye images, both an adjustment distance and a representative velocity were estimated. Specifically, the initial dye patch radius $L_{d}$ was estimated by averaging the radius of 2 concentric circles shortly after the dye was injected, whereas $L_{d}$ was estimated after approximately 2 inertial periods after an eddy had formed (panels 2 and 5 of Fig. 6). Differencing these two values, for the experiment shown we obtained an adjusted radius of approximately $\Delta L=8.8 \pm 1.6 \mathrm{~cm}$, where the difference between the radii of two concentric circles in the relevant subpanels was used to estimate the uncertainty.

Meanwhile, to estimate angular displacement and hence adjusted velocity $U_{\max }$, distinct features in the dye images were tracked for 1-2 inertial periods (e.g., panels 4 and 6 of Fig. 6) and used to estimate an angular velocity and radial location, giving a tangential velocity. Multiple such measurements were made to estimate, from the dye, $U_{\max }$ for each experiment. For these measurements, uncertainties were computed from the relative error averaged over all the experiments, with standard deviation taken as the absolute error for each experiment. This yielded a mean normalized uncertainty estimate of $24 \%$.

Regarding the dye images shown in Fig. 6, a few points merit further mention. The first is the asymmetry seen in the dye patch at later times in its evolution. We believe this is at least in part the result of small-scale motions driven by the grid mixer, which in practice are not negligible compared to the overall scale of the mixed patch. In fact, from panel 1 of Fig. 6, they are on the order of $1 / 10$ the size of the overall mixed patch, consistent with the grid mixer having of order 10 wires/grid cells across its diameter. These small features, evident in the first few panels of Fig. 6, eventually coalesce into the larger features evident at later times; the latter, being fewer in number, appear as asymmetries. Regarding the significance of these asymmetries, we note that the dye is not a perfect marker of the adjusting vortices: that is, not all fluid involved in the adjustment is marked with dye. By the nature of how the mixed patch was created, there are inevitably some regions of fluid near the perimeter of the grid mixer that mix without dye (recall the dye was injected only at the center of the grid mixer). These perimeter regions are thus also part of the adjustment (but without dye) after the grid mixing has stopped. This is 


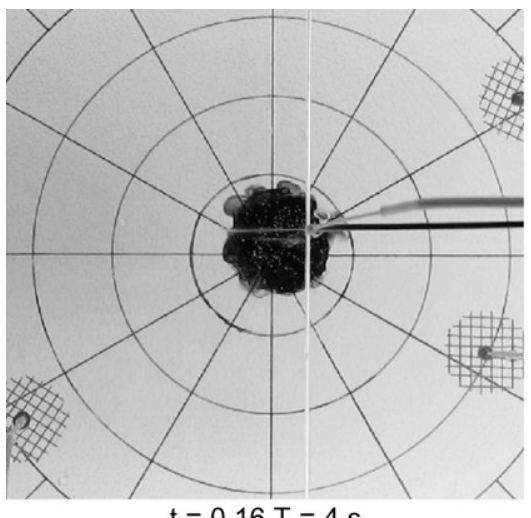

$t=0.16 T=4 \mathrm{~s}$

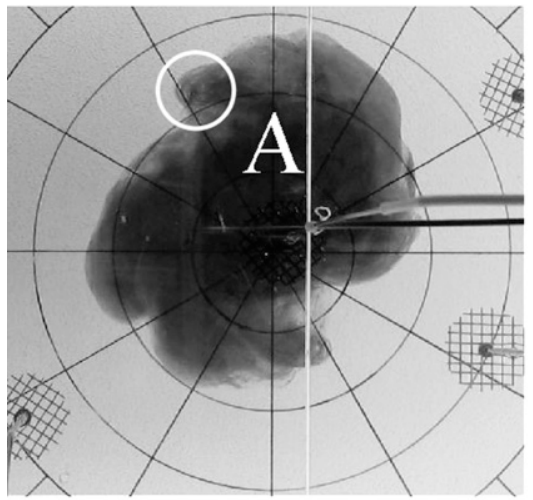

$t=1.39 T=35 s$

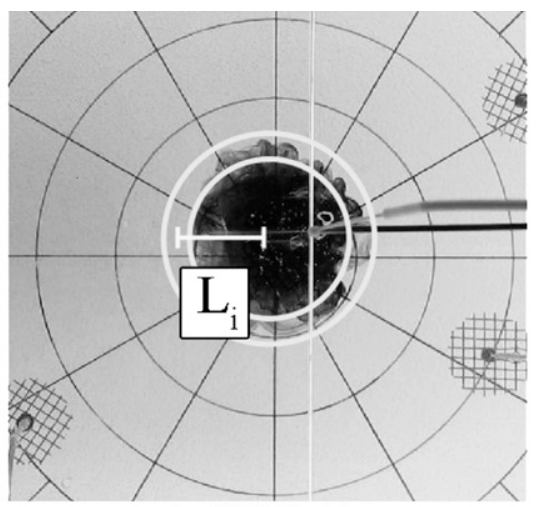

$\mathrm{t}=0.28 \mathrm{~T}=7 \mathrm{~s}$

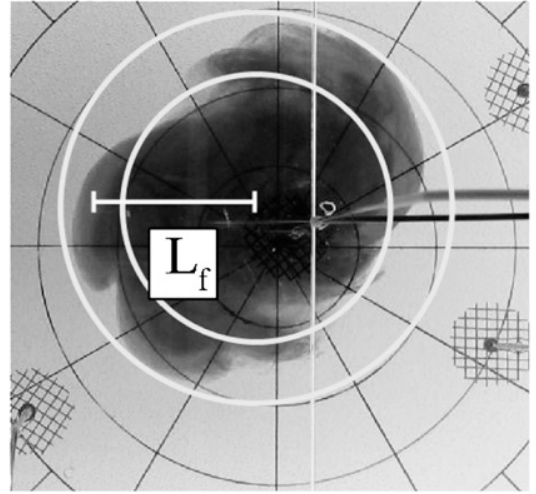

$t=1.83 T=46 s$

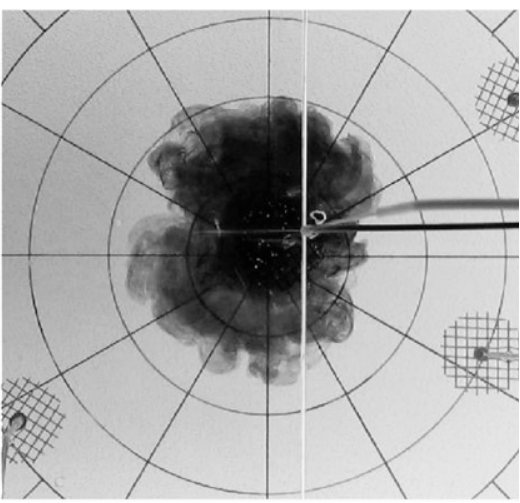

$\mathrm{t}=0.64 \mathrm{~T}=16 \mathrm{~s}$

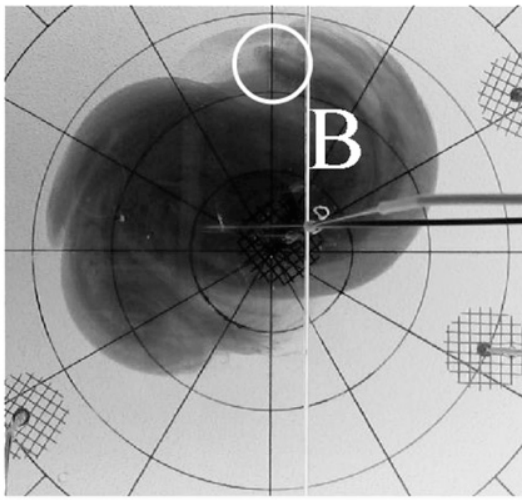

$\mathrm{t}=2.59 \mathrm{~T}=65 \mathrm{~s}$

FIG. 6. Time evolution of geostrophic adjustment in plan view. Concentric white circles indicate estimates of the minimum and maximum extent of the mixed patch at $t=7 \mathrm{~s}$ and of the adjusted eddy radius at $t=46 \mathrm{~s}$. The start (point $\mathrm{A}$ ) and end (point $\mathrm{B}$ ) positions used to estimate the velocity of a wisp located along the perimeter of the patch are marked by circles near A and B. Background grid is drawn at 5 -cm increments radially and $30^{\circ}$ azimuthally. Note the variable time between successive images.

borne out by the PIV measurements described in the next section, which shows no systematic asymmetries in the eddy velocities. In short, the dye should be viewed only as an approximate indicator of the eddy locationsize, and we have attempted to reflect this in the above stated uncertainties of the dye-derived measurements of $\Delta L$ and $U_{\max }$.

\section{c. $\Delta L$ and $U_{\max }$ from PIV measurements}

Additional independent estimates of the adjustment distance $\Delta L$ and adjusted velocity $U_{\max }$ were made using PIV. For this, a second set of five experiments was run using the same parameters as in the dye runs, except that the fluid was seeded with neutrally buoyant reflective particles prior to each fill rather than being injected with dye. The PIV system consisted of a twin-cavity neodymiumdoped yttrium aluminum garnet crystal (Nd:YAG) laser and two LaVision FlowMaster charge-coupled device (CCD) cameras equipped with bandpass filters to detect only the laser wavelength. A 2.0-mm horizontal laser light sheet illuminated the target depth at $d=15 \mathrm{~cm}$ : that is, the mid stroke depth of the grid mixer (see Fig. 4). PIV image pairs were taken 10 times per table rotation for 20-30 inertial periods. Image analysis was performed using the DaVis 7.1 PIV software (LaVision, GmbH 2005).

For the base case PIV run, velocity fields associated with the adjustment and spinup of a coherent eddy are shown in Fig. 7. Initially, the turbulent action of the mixer can be seen for $t<0$ (the time before the mixer stops), followed by the formation of a stable coherent eddy and its subsequent frictional decay. Notable in the later images is a $5-\mathrm{cm}$ region of near- 0 velocity at the center of the adjusted eddy. This is the result of contamination in the PIV analysis caused by the motionless grid mixer. Note, however, that we minimize the effect of the stopped mixer on the actual vortex velocities by "parking" the mixer above the core anticyclone after the initial mixing has stopped.

For all PIV runs, both the radius of maximum velocity and the maximum velocity itself $U_{\max }$ were estimated directly from the PIV fields for each time snapshot. For 

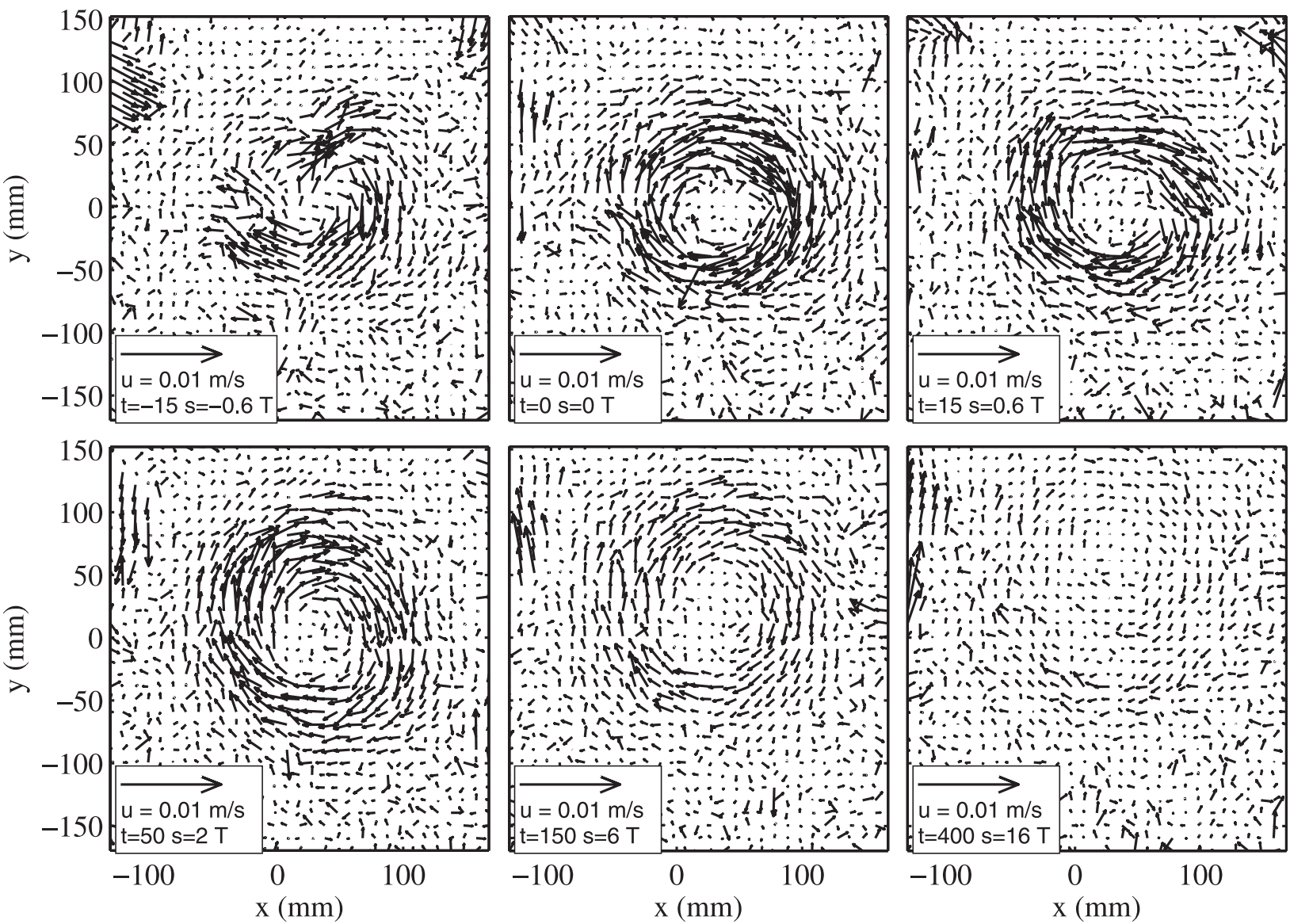

FIG. 7. Example of geostrophic adjustment based on 2D PIV image time series from a base run experiment $\left(f=0.25 \mathrm{rad} \mathrm{s}^{-1}\right.$ and $\Delta N=0.86 \mathrm{rad} \mathrm{s}^{-1}$ ). Note the variable time between successive images.

the radius of maximum velocity, the eddy radius was taken as the average distance from the eddy center to each of the $x, y$ components of maximum velocity. The resulting eddy radii were then averaged over the first two inertial periods following the mixer shutoff to obtain the average radius of maximum velocity for the experiment. Coincident with this analysis, the azimuthally averaged maximum velocity was also estimated by averaging the azimuthal velocity in a band $\Delta r= \pm 0.5 \mathrm{~cm}$ about the estimated radius of maximum velocity. As with the radius, the time average over the first two inertial periods following mixer shutoff was used to obtain the final value of $U_{\max }$.

The time evolution of the radius of maximum velocity and its value over the course of the adjustment for the base case run are shown in Fig. 8. Noteworthy is the clear exponential decay with time of the eddy velocity and corresponding increase in the eddy radius. The $e$-folding time scale estimated using an exponential fit to this decay was $264 \mathrm{~s}$ or about 10.5 inertial periods. Given the vertical scale of the eddy, this is about 10 times faster than would be expected because of molecular viscosity.
Whether this is due to internal wave radiation, the presence of the grid mixer ultimately retarding the flow or other processes is not known. However, a key point is that the Ekman number associated with the vortex is still small, so that to lowest order the mixed patch adjustment is geostrophic.

Although not a direct measurement of the adjustment distance $\Delta L$, the radius of maximum velocity is also related to the deformation radius $R$. The radius of maximum velocity thus provides an independent estimate of the horizontal scale of the adjustment. For the base case PIV run, from the time series shown in Fig. 8, we obtain a radius of maximum velocity of $5.2 \pm 0.5 \mathrm{~cm}$, which was slightly less than the adjustment length estimated from the dye method $\Delta L=8.8 \mathrm{~cm}$. Meanwhile, also for the base case, the maximum velocity estimated using PIV was $U_{\max }=0.26 \pm 0.07 \mathrm{~cm} \mathrm{~s}^{-1}$, compared to $U_{\max }=0.24 \mathrm{~cm} \mathrm{~s}^{-1}$ estimated from the dye; that is, these 2 estimates were equal to within measurement uncertainty.

An additional feature worth noting in the time series in Fig. 8 are the smaller-scale fluctuations superimposed 

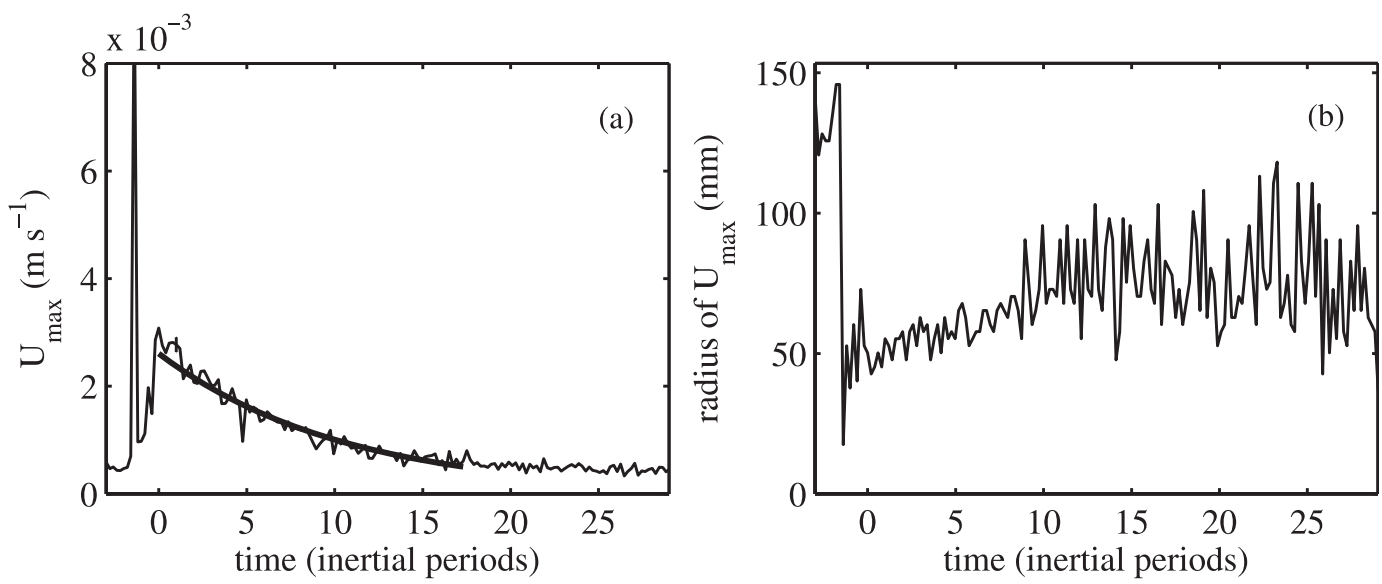

FIG. 8. Time series of (a) the maximum azimuthal velocity $U_{\max }$ and (b) the radius at which it occurred. Thick solid curve in (a) is an exponential fit to the velocity decay, corresponding to an $e$-folding time scale of $264 \mathrm{~s}$ or 10.5 inertial periods. In both (a) and (b), $t=0$ corresponds to the end of the mechanical mixing and the beginning of the adjustment.

on the longer time-scale exponential decay. These fluctuations as well as clear evidence in our numerical experiments (not shown) suggest the presence of internal waves, including near-inertial oscillations, radiating from the mixed region during the adjustment. It is tempting to consider the relationship that these oscillations may have, for example, to the pulson solutions described in the literature (e.g., Cushman-Roisin 1987; Rubino and Brandt 2003). The radiation of waves during the adjustment is discussed in more detail by Lelong and Sundermeyer (2005) in the context of the numerical solutions. However, in the present study, we are more interested in the balanced state of the resulting vortex. Because our analysis of velocities from the laboratory experiments averages over one or more inertial periods, such waves are averaged out of our final results. Also, the evidence for such oscillations in the laboratory analyses presented here is tenuous at best; the time series of maximum velocity in Fig. 8, for example, shows only subtle oscillations, not all of which are inertial, over the lifetime of the eddy.

Returning to the full PIV fields shown in Fig. 7 again, a few points merit further discussion. First, we noted that we use two CCD cameras to create stereographic images of particles within the laser sheet. This allows us to compute three-dimensional velocities for each PIV snapshot. In our analysis, however, we are most interested in the horizontal velocities associated with the adjusting mixed patch. As such, our primary purpose for using 3D PIV is to avoid having vertical velocities aliased into our horizontal velocity estimates, as can occur with single camera PIV observations. Nevertheless, the vertical velocities and hence the horizontal velocities as well are still prone to errors inherent in the measurement technique.
Postprocessing of the PIV velocity estimates attempts to remove spurious velocities using critical threshold criteria as well as median filtering. However, inevitably the measurements still contain errors and spurious data.

Relating this to our PIV-derived velocities of Fig. 7, we note that the large velocities in and around the mixer in the first figure panel are likely a combination of real horizontal velocities and vertical velocities aliased into the horizontal velocity signal. What effect these vertical velocities ultimately have on the developing vortex is not known and is beyond the scope of this study; again, we focus here only on the eventual balanced eddy. However, we do note that, despite the presumably large vertical velocities early in the experiment, the horizontal velocities appear to organize themselves rather rapidly into a coherent eddy after a remarkably short time, on the order of an inertial period.

Regarding the localized regions of high velocities near the top-left corner of the various panels in Fig. 7, we note that this region is neither near the tank boundary nor near any other structure or feature within the tank. Given the localized nature of this anomalous velocity, plus the fact that it changes/reverses direction multiple times over the course of the experiment, we believe it is a result of the PIV measurement technique and associated processing and not a real feature. That this region repeatedly showed spurious velocities may, for example, be the result of a poor calibration of the PIV grid and associated corrections in that portion of the domain.

Finally, in all of the above, we note that the way in which we use the PIV data, identifying the radius of maximum velocity based on both $x$ - and $y$-velocity profiles and azimuthally averaging to obtain the maximum eddy velocity, means that localized velocity errors, particularly 
TABLE 1. Summary of parameter values for laboratory experiments.

\begin{tabular}{|c|c|c|c|c|c|c|}
\hline Expt & PIV or dye & $f\left(\operatorname{rad~s}^{-1}\right)$ & $\Delta N\left(\operatorname{rad~s}^{-1}\right)$ & $\mathrm{Bu}^{1 / 2}(R / L)$ & $\Delta L$, radius of $U_{\max }(\mathrm{cm})$ & $U_{\max }\left(\mathrm{cm} \mathrm{s}^{-1}\right)$ \\
\hline \multirow[t]{2}{*}{ Base run } & dye & 0.25 & 0.86 & 4.3 & 8.8 & 0.24 \\
\hline & PIV & 0.25 & 0.86 & 4.3 & 5.2 & 0.26 \\
\hline \multirow[t]{2}{*}{$2 f$} & dye & 0.50 & 0.86 & 2.1 & 4.1 & 0.33 \\
\hline & PIV & 0.50 & 0.86 & 2.1 & 4.9 & 0.36 \\
\hline \multirow[t]{2}{*}{$0.5 f$} & dye & 0.125 & 0.86 & 8.6 & 8.9 & 0.24 \\
\hline & PIV & 0.125 & 0.86 & 8.6 & 7.1 & 0.11 \\
\hline \multirow[t]{2}{*}{$2 \Delta N$} & dye & 0.25 & 1.72 & 8.6 & 7.4 & 0.52 \\
\hline & PIV & 0.25 & 1.72 & 8.6 & 6.5 & 0.41 \\
\hline \multirow[t]{2}{*}{$2 \Delta N, 2 \Delta f$} & dye & 0.50 & 1.72 & 4.3 & 5.3 & 0.93 \\
\hline & PIV & 0.50 & 1.72 & 4.3 & 5.1 & 0.66 \\
\hline
\end{tabular}

ones outside the main eddy, have little effect on our analysis and hence our main conclusions.

\section{d. Comparison with theoretical parameter dependence}

Using the above described experiment as a base and following the same analysis techniques, experiments were repeated but varying the buoyancy frequency $N$ and the rotation rate $f$ by a factor of 2 up or down compared to the base case. Specifically, experiments were run doubling and halving $f$ holding $\Delta N$ constant, doubling $\Delta N$ holding $f$ constant, and doubling both $\Delta N$ and $f$. These initial conditions thus resulted in three $R / L$ ratios, namely $2.1,4.3$, and 8.6 , corresponding to a variation in $\mathrm{Bu}$ of a factor of 16 . The initial parameters for each experiment and the observed velocities and adjustment lengths are listed in Table 1. The initial parameters and observed values for both the PIV and dye base run experiments are also listed in the table for comparison.

The observed adjustment distances $\Delta L$ and maximum adjusted velocities $U_{\max }$ for the 10 laboratory experiments listed in Table 1 are plotted against their corresponding $R / L$ ratios in Figs. 2 and 3, along with the analytical and numerical results. As noted above, the dye and PIV estimates of the adjustment distances differed somewhat for the base run. This can be seen in Fig. 2, where for $R / L=4.3$ the base case dye estimate (i.e., the triangle closest to the M88 curve) is distinctly higher than the other three measurements for the same $R / L$ value (note that these three points appear as one because their values are almost identical). Note, however, that the other observed PIV and dye adjustment lengths for $R / L=4.3$, corresponding to both $\Delta N$ and $f$ being doubled, were equal to within measurement uncertainty (i.e., radius of $U_{\max }=5.1 \pm 0.3 \mathrm{~cm}$ from PIV versus $\Delta L=5.3 \pm 1.8 \mathrm{~cm}$ from dye). Similarly, the observed adjusted velocities for both dye and PIV are shown in Fig. 3 plotted as a function of corresponding $R / L$ ratios for each run. Particularly noteworthy is the rolloff with increasing $R / L$ for $\mathrm{Bu}>1$, in agreement with both the analytical and numerical solutions.

\section{Discussion}

Analytical solutions of the adjustment of an isolated lens reveal key differences at large and small Burger number between solutions stemming from different but closely related initial condition geometries. Considering radial displacements and maximum velocities, the different initial conditions examined here can be grouped into three categories based on three defining factors. First, the 2D layered dam break problem, described by Gill (1982), is unique in that its initial condition contains only one inherent length scale, the deformation radius $R$. For this case, the maximum adjusted velocity $U_{\max }$ corresponds to the reduced-gravity wave speed $\sqrt{g^{\prime} H}$, whereas the adjustment distance $\Delta L$ corresponds exactly to the deformation radius $R$ (the latter can also be interpreted as the advective length scale with $1 / f$ as the time scale of advection and $U_{\max }$ as the relevant velocity; i.e., $\left.\Delta L=U_{\max } / f=\sqrt{g^{\prime} H} / f=R\right)$. Second are the tophat solutions of Csanady (1979) and Flierl (1979), which contain a second length scale, the finite size of the density perturbation $L_{d}$. This has the effect of modifying the solution for $\mathrm{Bu}>1$, such that both the adjustment distance $\Delta L$ and the adjusted velocity scale $U_{\max }$ are reduced compared to the case of infinite $L_{d}$. This reduction can be attributed to the finite volume of the initial anomaly, which leads to a decrease in $h$ (and hence the effective $R$ ) as the horizontal scale of the density defect $L_{d}$ increases (slumps) during the adjustment. Third, the layered parabolic and continuously stratified lens solutions of M88 contain a third (nonzero) length scale associated with the initial pressure gradient $L_{p}$. For large $\mathrm{Bu}$, by virtue of the finite horizontal scale of the initial density defect $L_{d}$, these cases behave in a similar manner as the second case. However, for small $\mathrm{Bu}$, they exhibit a decrease in both the adjustment distance $\Delta L$ and the adjusted velocity $U_{\max }$ compared to the first two scenarios. 
This decrease at small $\mathrm{Bu}$ can be attributed to the reduced pressure gradient compared to the previous cases: that is, one that occurs over a finite scale rather than as a delta function corresponding to a density discontinuity. Noteworthy in this third case is that, for small $\mathrm{Bu}$, the maximum adjusted velocity $U_{\max }$ is exactly equal to that obtained from pressure gradient scaling, $U_{\max }=N^{2} h^{2} / f L$, whereas the adjustment distance $\Delta L$ is again equal to the corresponding advective length scale with $1 / f$ as the time scale of advection: that is, $\Delta L=U_{\max } / f=\left(N^{2} h^{2} / f L\right) / f=$ $R^{2} / L$ or $\Delta L / R=R / L$.

For the case of an isolated lens in a continuously stratified fluid, comparing the analytical solutions to the numerical solutions, we find that both the adjustment distance $\Delta L$ and the maximum adjusted velocity $U_{\max }$ bear out the large and small $\mathrm{Bu}$ dependence seen in the analogous analytical solutions. Despite the much more limited parameter range spanned by the laboratory experiments, they too are consistent with the predicted rolloff for large Burger numbers. Particularly noteworthy in the latter is the simple point that, even for $\mathrm{Bu} \gg 1$, the adjustment ultimately results in a geostrophically balanced state, albeit with scales that are considerably different than simple scaling based on initial conditions might suggest. That the adjusted state is indeed balanced can has been confirmed in the numerical solutions by computing geostrophic velocities from the model pressure field and comparing them to the actual velocities in the model (not shown). Also noteworthy here is that both our laboratory simulations and our numerical results allow for nonhydrostatic effects. This notwithstanding, aside from the initial radiation of internal waves associated with geostrophic adjustment, the motions associated with the vortices themselves are approximately hydrostatic, because vertical velocities are relatively small in the balanced state of the vortex (e.g., see also Dritschel and Viúdez 2007). The key point here, however, is that, even for $\mathrm{Bu} \gg 1$, the adjustment can still proceed to a stable, geostrophically balanced state, provided the effect of friction (i.e., the Ekman number) is small.

The latter point also raises the question of how the behavior depicted in Figs. 2 and 3 would be modified in the presence of frictional effects. Regarding $\Delta L$, regardless of Burger number, we expect the radial adjustment distance to be greater than the geostrophically constrained case because $R$ would no longer be a limiting length scale. This assumes, of course, that the density anomaly is not first dissipated away by diffusive processes. In terms of $U_{\max }$, we would expect the azimuthal velocity associated with the balanced part of the motion to be reduced compared to the geostrophic adjustment case, because, in the frictional case, a portion of the kinetic energy would be tied up in the radial motion and the azimuthal motion itself would be reduced by friction. Of course, in the frictional case, the adjustment would also not achieve a steady state until all motion had ceased.

Noteworthy regarding the analytical solutions depicted in Figs. 2 and 3 is the somewhat muted effect that the inclusion/exclusion of nonlinear terms has on the final adjusted solution in terms of $\Delta L$ and $U_{\max }$ when compared to the variations due to $\mathrm{Bu}$ dependence. Although a twofold to fourfold difference in the two-dimensional versus three-dimensional scenarios is certainly an order one effect, this pales compared to the more than order of magnitude differences for $\mathrm{Bu} \gg 1$ and $\mathrm{Bu} \ll 1$ seen between the step function solution (e.g., Gill 1982; Cushman-Roisin 1994) and the localized continuous pressure gradient solutions M88.

Also noteworthy for the continuously stratified solution of M88, as well as the numerical and laboratory results presented here, is that the Rossby number U/fL of the adjusted state is always less than 1 (see Fig. 3). This follows directly from gradient wind balance for high pressure anticyclonic eddies. As noted by Olson (1991) in his survey of ocean rings, anticyclones in general may thus be less nonlinear and hence potentially more stable than cyclonic eddies. Note also that the vortices in the present study are "shielded" vortices in the sense that they consist of an anticyclonic core surrounded by a ring of positive vorticity. As discussed, for example, by Kloosterziel and Carnevale (1999), the stability of such vortices depends not only on whether the vorticity changes sign but also on the steepness of the vorticity profile with respect to the radial coordinate: the steeper the vorticity profile, the faster the instability growth rate for higher wavenumbers. Thus, steeper vorticity profiles successively lead to the formation of dipoles, tripoles, and higher mode structures such as those reported by Rubino et al. (2002) and elsewhere in the literature. Conversely, shallower vorticity profiles have longer instability growth times and hence are more stable.

Putting the above in the context of the present study, we believe the vortices examined here are comfortably in the regime of being stable with relatively slow instability growth rates. This is evidenced by the fact that in both the laboratory and our numerical simulations our vortices are remarkably coherent and symmetric for a considerable time: at least 10 inertial periods (the $e$-folding decay time scale of our eddies) in the laboratory and hundreds of inertial periods in the model. That said, we have conducted additional numerical investigations using the same numerical model and have found that indeed, when seeded with noise, our vortices do eventually become unstable (i.e., after hundreds of inertial periods; results not shown). In the absence of other 
forcing, we believe this instability ultimately is the result of barotropic instability, because the growth time is consistent with theoretical predictions for this process. Conversely, when superimposed on larger-scale background vertical shears, we find that our vortices go unstable more quickly, with grow rates in that case more consistent with those expected for baroclinic instability. That the eventual instability of the unforced eddies is barotropic rather than baroclinic is similar to findings of Beckers et al. (2003) for shielded vortices in a nonrotating fluid. A more in depth treatment of the stability of $\mathrm{s}$ vortices is a subject for ongoing investigation.

Stability aside, a significant result of the present study is the simple fact that results from both numerical simulations and laboratory experiments were in good agreement with semianalytical solutions by M88 for the adjustment of a continuously stratified isolated lens for a wide range of $\mathrm{Bu}$. In all cases, an axisymmetric lens beginning from a state of rest was allowed to adjust under the influence of gravity and rotation to form a geostrophically balanced eddy. Regarding adjustment distances, the laboratory results were slightly larger than corresponding numerical and analytical solutions. However, they were only between a factor of 1 to 3 larger: that is, consistent to within an order 1 scale factor. Meanwhile, the maximum adjusted velocity $U_{\max }$ exhibited strong $\mathrm{Bu}$ dependence in both the numerical and laboratory simulations, in close agreement with the M88 solutions. Most importantly, however, was that both the numerical and laboratory results, as well as the analytical solutions of M88, all differed significantly from geostrophic scaling based on the parameters of the initial mixed patch. In fact, the solutions for $U_{\max }$ were as much as two orders of magnitude smaller than geostrophic scaling in the $\mathrm{Bu} \gg 1$ regime where the laboratory experiments were conducted, despite the experiments clearly resulting in a geostrophically balanced end state. Any reasonable prediction of the adjusted state, particularly for $\mathrm{Bu}$ significantly different from unity, must take this into account

Finally, regarding the laboratory experiments, a limitation of the present study is that less than an order of magnitude range of $R / L$ ratios was spanned in the present experiments (see data shown in Figs. 2,3) because of constraints of the experimental setup. In particular, $\mathrm{Bu}<1$ could not be reached because of a number of reasons. First, the 1.0-m-diameter tank size limited how large the initial mixed patch could be without feeling sidewall effects. Also, the value of $R$ was constrained by $\Delta N, h$, and $f$. That is, the value of $h$ was already small and hence constrained by the Ekman number so as to avoid frictional effects. Any substantial decrease in $h$ would cause the Ekman number to approach unity, thereby changing the dynamics of the adjustment. Meanwhile, increasing $f$ or decreasing $N$ further would have brought these two time scales too close together and, for the aspect ratio of the present experiments (i.e., $h / L$ ), would have approached an unstable eddy regime (e.g., Stegner et al. 2004).

\section{Summary and conclusions}

In this study we revisited the problem of geostrophic adjustment of an isolated lens in a stratified fluid. A progression of published analytical solutions for the Rossby adjustment problem was reexamined for initial conditions ranging from a $2 \mathrm{D}$ dam break to a continuously stratified axisymmetric lens. These solutions were then compared to numerical simulations and laboratory experiments of the adjustment of an isolated lens in a continuously stratified rotating environment. Major results of this study are as follows.

Using a progression of analytical solutions, we identified the roles of three distinct length scales associated with the adjustment problem, the Rossby radius of deformation $R$, the length scale associated with the density defect $L_{d}$, and the length scale associated with the pressure gradient $L_{p}$. For $\mathrm{Bu} \ll 1$, the adjusted velocity approaches one of two solutions, depending on the geometry of the initial condition. When no initial length scale of the pressure gradient is defined (i.e., density is a step function such that $L_{p}=0$ ), velocity scales as the reduced-gravity wave speed, whereas the adjustment distance $\Delta L$ scales as the advective length scale associated with an inertial time scale (i.e., $\Delta L=U_{\max } / f$ ). When the initial pressure gradient scale $L_{p}$ is nonzero, velocity scales according to geostrophic pressure gradient scaling via the momentum equations, whereas the adjustment distance $\Delta L$ again scales as $\Delta L=U_{\max } / f$, this time with $U_{\max }$ given by geostrophic scaling. For $\mathrm{Bu} \gg 1$, all solutions with finite $L_{d}$ scale such that both the geostrophically balanced velocity and the adjustment length scale are less than the reduced-gravity wave speed and its associated advective length scale. This reduction can be attributed to volumetric effects, which reduce the effective pressure gradient during the adjustment and hence limit both the velocity and the displacement by an amount proportional to Bu. Noteworthy is that differences between the respective solutions can be manyfold, up to an order of magnitude, as Bu becomes either large or small. This highlights the importance of properly identifying and estimating the relevant length scales when predicting the adjusted state based on initial conditions.

Regarding the initial and final states, we note that the final solutions can achieve geostrophically balanced states even when Bu based on initial parameters is large. This 
is because, during the adjustment, the initial slumping of the density defect modifies both the vertical and horizontal scales of the density defect such that, as $L$ increases, $h$ decreases, leading to a decrease in the horizontal pressure gradient. Thus, the effective deformation radius decreases, whereas the relevant horizontal length scale of the density defect increases. The adjustment itself thus causes the $\mathrm{Bu}$ of the adjusted state to approach unity. Presumably, however, this can only occur if viscosity or diffusion is not so large that it limits the adjustment before rotation becomes important.

Considering the numerical simulations, we found that for a continuously stratified isolated lens, the end state computed from the fully nonlinear time-dependent solution agreed both qualitatively and quantitatively with the analytical solution of M88 for the same geometry. Although the behavior of such numerical solutions was examined in detail by Lelong and Sundermeyer (2005), the interpretation in the context of other related initial geometries and in terms of the Burger number dependence of adjustment distance and adjusted velocity is new to the present study.

Finally, regarding the laboratory experiments, we find that experiments conducted at large $\mathrm{Bu}$ are consistent with both numerical and analogous analytical solutions for an axisymmetric lens in terms of adjustment distance and maximum adjusted velocity. To our knowledge, this is the first time that laboratory results using this form of initial condition have been reported in the literature (i.e., the generation of a mixed patch in a continuously stratified rotating fluid via mixing, as opposed to mass injection or direct velocity forcing).

Relevant to all of the analyses presented here is that the dynamics of geostrophic adjustment can be applied to a variety of scales, from mesoscale to submesoscale, by appropriately rescaling the relevant nondimensional parameters. At one end of the spectrum is the context envisioned by Csanady (1979) and Flierl (1979) (i.e., Mediterranean eddies, or meddies). At intermediate scales, M88 relates his solutions to the problem of submesoscale coherent vortices on the order of $10 \mathrm{~km}$ horizontally and $100 \mathrm{~m}$ vertically. Finally, at still smaller scales, Lelong and Sundermeyer (2005) and Sundermeyer and Lelong (2005) relate their findings to mixed patches generated by internal wave breaking on scales of hundreds of meters to a few kilometers horizontally and on the order of $0.5-10 \mathrm{~m}$ vertically. In all of these cases, an effective deformation radius can be clearly defined, and the adjustment can be interpreted in the context of geostrophic adjustment. As evidenced by the present laboratory experiments in particular, even at very small scales and for large Bu based on initial conditions, geostrophic adjustment can still occur as long as friction does not dominate.
Acknowledgments. Laboratory experiments and their interpretation were supported by the National Science Foundation under Grants OCE-0351892 and OCE0351905. Numerical modeling work was supported in part by the Office of Naval Research under Grant N00014-011-0984 and by the National Science Foundation under Grant OCE-0623193. Jim Fontaine provided engineering assistance for the laboratory experiments. Chris Luebke assisted with the initial design and fabrication of the laboratory mixers. Geoff Cowles assisted with the calculation of seminumerical solutions for the continuously stratified solutions from the published literature. We also thank two anonymous reviewers for helpful suggestions and feedback.

\section{REFERENCES}

Alford, M. A., and R. Pinkel, 2000: Observations of overturning in the thermocline: The context of ocean mixing. J. Phys. Oceanogr., 30, 805-832.

Armi, L., and W. Zenk, 1984: Large lenses of highly saline Mediterranean water. J. Phys. Oceanogr., 14, 1560-1576.

Australian Scientific Instruments, cited 2010: The ANU rotating table. Australian National University Research School of Earth Sciences. [Available online at http://rses.anu.edu.au/gfd/index. php? $\mathrm{p}=$ table.]

Bartello, P., 1995: Geostrophic adjustment and inverse cascades in rotating stratified turbulence. J. Atmos. Sci., 52, 4410-4428.

Beckers, M., H. Clercx, G. van Heijst, and R. Verzicco, 2003: Evolution and instability of monopolar vortices in a stratified fluid. Phys. Fluids, 15, 1033-1045.

Blumen, W., 1972: Geostrophic adjustment. Rev. Geophys. Space Phys., 10, 485.

— energy conversion. J. Phys. Oceanogr., 25, 428-438.

Boss, E., and L. Thompson, 1995: Energetics of nonlinear geostrophic adjustment. J. Phys. Oceanogr., 25, 1521-1529.

Cenedese, C., J. Whitehead, T. Ascarelli, and M. Ohiwa, 2004: A dense current flowing down a sloping bottom in a rotating fluid. J. Phys. Oceanogr., 34, 188-203.

Charney, J., 1971: Geostrophic turbulence. J. Atmos. Sci., 28, 10871094.

Csanady, G., 1979: The birth and death of a warm core ring. J. Geophys. Res., 84 (C2), 777-780.

Cushman-Roisin, B., 1987: Exact analytical solutions for elliptical vortices of the shallow-water equations. Tellus, 39A, 235-244.

_ 1994: Introduction to Geophysical Fluid Dynamics. Prentice Hall, 320 pp.

D'Asaro, E., 1988: Generation of submesoscale vortices: A new mechanism. J. Geophys. Res., 93 (C6), 6685-6693.

De Silva, I., and H. Fernando, 1998: Experiments on collapsing turbulent regions in stratified fluids. J. Fluid Mech., 358, 29-60.

Dewar, W., and P. Killworth, 1990: On the cylinder collapse problem, mixing, and the merger of isolated eddies. J. Phys. Oceanogr., 20, $1563-1575$.

Dotsenko, S., and A. Rubino, 2006: Analytical solutions for circular stratified eddies of the reduced-gravity shallow-water equations. J. Phys. Oceanogr., 36, 1693-1702.

Dritschel, D., and A. Viúdez, 2007: The persistence of balance in geophysical flows. J. Fluid Mech., 570, 365-383. 
Flierl, G. R., 1979: A simple model for the structure of warm and cold core rings. J. Geophys. Res., 84 (C2), 781-785.

_ 1987: Isolated eddy models in geophysics. Annu. Rev. Fluid Mech., 19, 493-530.

Fortuin, J., 1960: Theory and application of two supplementary methods of constructing density gradient columns. J. Polym. Sci., 44, 505-515.

Garrett, C., and W. Munk, 1972: Oceanic mixing by breaking internal waves. Deep-Sea Res., 19, 823-832.

Garvine, R., 1987: Estuary plumes and fronts in shelf waters: A layer model. J. Phys. Oceanogr., 17, 1877-1896.

Geisler, J., 1970: Linear theory of the response of a two layer ocean to a moving hurricane. Geophys. Astrophys. Fluid Dyn., 1, 249-272.

Gill, A., 1982: Atmosphere-Ocean Dynamics. Academic Press, 662 pp.

Grant, H. L., A. Moilliet, and W. M. Vogel, 1968: Some observations of the occurrence of turbulence in and above the thermocline. J. Fluid Mech., 34, 443-448.

Gregg, M., 1980: Microstructure patches in the thermocline. J. Phys. Oceanogr., 10, 915-943.

_ persistent mixing and near-inertial internal waves. J. Phys. Oceanogr., 16, 856-885.

Hebert, D., N. Oakey, and B. Ruddick, 1990: Evolution of a Mediterranean salt lens: Scalar properties. J. Phys. Oceanogr., 20, $1468-1483$

Hedstrom, K., and L. Armi, 1988: An experimental study of homogeneous lenses in a rotating, stratified fluid. J. Fluid Mech., 191, 535-556.

Hermann, A., and W. Owens, 1993: Energetics of gravitational adjustment for mesoscale chimneys. J. Phys. Oceanogr., 23, 346-371.

Hopfinger, E., and G. van Heijst, 1993: Vortices in rotating fluids. Annu. Rev. Fluid Mech., 25, 241-289.

Killworth, P., 1979: On "chimney" formations in the ocean. J. Phys. Oceanogr., 9, 531-554.

_ 1992: The time-dependent collapse of a rotating fluid cylinder. J. Phys. Oceanogr., 22, 390-397.

Kloosterziel, R., and G. Carnevale, 1999: On the evolution and saturation of instabilities of two-dimensional isolated circular vortices. J. Fluid Mech., 388, 217-257.

Kunze, E., 2001: Waves: Vortical mode. Encyclopedia of Ocean Sciences, S. T. J. Steele and K. Turekian, Eds., Academic Press, 3174-3178.

- and T. Sanford, 1993: Submesoscale dynamics near a seamount. Part I: Measurements of Ertel vorticity. J. Phys. Oceanogr., 23, 2567-2601.

Kuo, A., and L. Polvani, 1997: Time-dependent fully nonlinear geostrophic adjustment. J. Phys. Oceanogr., 27, 1614-1634.

LaVision, GmbH, 2005: DaVis Flowmaster software manual. 184 pp.

Lelong, M., and M. Sundermeyer, 2005: Geostrophic adjustment of an isolated diapycnal mixing event and its implications for small-scale lateral dispersion. J. Phys. Oceanogr., 35, 2352-2367.

Marshall, J., A. Nurser, and R. Williams, 1993: Inferring the subduction rate and period over the North Atlantic. J. Phys. Oceanogr., 23, $1315-1329$.

McWilliams, J. C., 1985: Submesoscale, coherent vortices in the ocean. Rev. Geophys., 23, 165-182.

_ 1988: Vortex generation through balanced adjustment. J. Phys. Oceanogr., 18, 1178-1192.

Morel, Y., and J. McWilliams, 1997: Evolution of isolated interior vortices in the ocean. J. Phys. Oceanogr., 27, 727-748.

Oakey, N. S., and B. J. W. Greenan, 2004: Mixing in a coastal environment: 2. A view from microstructure measurements J. Geophys. Res., 109, C10014, doi:10.1029/2003JC002193.
Olson, D., 1991: Rings in the ocean. Annu. Rev. Earth Planet. Sci., 19, 283-311.

Ou, H., 1984: Geostrophic adjustment: A mechanism for frontogenesis. J. Phys. Oceanogr., 14, 994-1000.

1986: On the energy conversion during geostrophic adjustment. J. Phys. Oceanogr., 16, 2203-2204.

Polzin, K. L., E. Kunze, J. M. Toole, and R. W. Schmitt, 2003: The partition of finescale energy into internal waves and subinertial motions. J. Phys. Oceanogr., 33, 234-248.

Price, J., 1981: Upper ocean response to a hurricane. J. Phys. Oceanogr., 11, 153-175.

-, and M. O'Neil Baringer, 1994: Outflows and deep water production by marginal seas. Prog. Oceanogr., 33, 161-200.

Reznik, G., V. Zeitlin, and M. Ben Jelloul, 2001: Nonlinear theory of geostrophic adjustment. Part 1. Rotating shallow-water model. J. Fluid Mech., 445, 93-120.

Robinson, A., M. Spall, and N. Pinardi, 1988: Gulf Stream simulations and the dynamics of ring and meander processes. J. Phys. Oceanogr., 18, 1811-1854.

Rossby, C., 1937: On the mutual adjustment of pressure and velocity distributions in certain simple current systems. J. Mar. Res., 1, 15-28.

_ 1938: On the mutual adjustment of pressure and velocity distribution in certain simple current systems. J. Mar. Res., 1, 239-263.

Rubino, A., and P. Brandt, 2003: Warm-core eddies studied by laboratory experiments and numerical modeling. J. Phys. Oceanogr., 33, 431-435.

, K. Hessner, and P. Brandt, 2002: Decay of stable warm-core eddies in a layered frontal model. J. Phys. Oceanogr., 32, 188-201.

- A. Androssov, and S. Dotsenko, 2007: Intrinsic dynamics and long-term evolution of a convectively generated oceanic vortex in the Greenland Sea. Geophys. Res. Lett., 34, L16607, doi:10.1029/2007GL030634.

Saunders, P., 1971: Anticyclonic eddies formed from shoreward meanders of the Gulf Stream. Deep-Sea Res., 18, 1207-1219. , 1973: The instability of a baroclinic vortex. J. Phys. Oceanogr., 3, 61-65.

Spall, M., 1995: Frontogenesis, subduction, and cross-front exchange at upper ocean fronts. J. Geophys. Res., 100 (C2), 2543-2557.

Stegner, A., P. Bouruet-Aubertot, and T. Pichon, 2004: Nonlinear adjustment of density fronts. Part 1 . The Rossby scenario and the experimental reality. J. Fluid Mech., 502, 335-360.

Stommel, H., 1979: Determination of water mass properties of water pumped down from the Ekman layer to the geostrophic flow below. Proc. Natl. Acad. Sci. USA, 76, 3051-3055.

Sundermeyer, M. A., and M.-P. Lelong, 2005: Numerical simulations of lateral dispersion by the relaxation of diapycnal mixing events. J. Phys. Oceanogr., 35, 2368-2386.

— J. R. Ledwell, N. S. Oakey, and B. J. W. Greenan, 2005: Stirring by small-scale vortices caused by patchy mixing. J. Phys. Oceanogr., 35, 1245-1262.

Ungarish, M., and H. Huppert, 1998: The effects of rotation on axisymmetric gravity currents. J. Fluid Mech., 362, 17-51.

van Heijst, G. J. F., 1985: A geostrophic adjustment model of a tidal mixing front. J. Phys. Oceanogr., 15, 1182-1190.

Whitehead, J., J. Marshall, and G. Hufford, 1996: Localized convection in rotating stratified fluid. J. Geophys. Res., 101, 25 705-25 722.

Winters, K. B., J. A. MacKinnon, and B. Mills, 2004: A spectral model for processes studies of rotating, density stratified flows. J. Atmos. Oceanic Technol., 21, 69-94.

Woods, J. D., and R. L. Wiley, 1972: Billow turbulence and ocean microstructure. Deep-Sea Res., 19, 87-121. 